\title{
Be a change agent: Tools and techniques to support organizational and individual transformation
}

\author{
Amy Gunty*, Jody Van Ness and Kelly Nye-Lengerman \\ Institute on Community Integration, University of Minnesota, Minneapolis, MN, USA
}

Revised/Accepted November 2018

\begin{abstract}
.
BACKGROUND: More people with disabilities are living and working in the community than ever before, including many who receive support from provider organizations and state agencies. These organizations play an important role in facilitating community living and employment. Organizational change is necessary as our system continues to shift away from segregating people with disabilities to a society in which people with disabilities are fully engaged and contributing their strengths and talents in their communities. However, there are well-established patterns of thinking, practice, and policy that make organizational change difficult to implement.

OBJECTIVE: This paper will describe lessons learned when working with a large agency to implement organization-wide adoption of person-centered practices (PCPs) using a model of episodic and continuous change (Quinn, 1996). This includes an overview of the episodic/continuous model of organizational change, with detailed information about how to implement each type of change.

CONCLUSIONS: Implementing well-balanced organizational change activities and strategies, including elements of episodic and continuous change, is an effective way to work toward lasting change within agencies that support people with disabilities in fully inclusive setting. This can be a useful tool to support increased competitive, integrated employment for people with disabilities.
\end{abstract}

Keywords: Competitive, integrated employment, intellectual and developmental disabilities, person-centered practices, organizational transformation, communities of practice

\section{Introduction}

Due to legislation, litigation, and a general social movement working for civil rights for people with disabilities, we are in a time when service organizations and other agencies that support people with disabilities (including intellectual and developmental disabilities [IDD]) are progressively and increasingly moving toward the adoption of person-centered practices (PCPs) and Employment First policies (Gunty,

\footnotetext{
*Address for correspondence: Amy Gunty, Institute on Community Integration; 204 Pattee Hall, 150 Pillsbury Dr. SE, Minneapolis, MN 55455, USA. Tel.: +1 612460 1782; E-mail: gunty004@umn.edu.
}

Dean, Nord, Hoff, \& Nye-Lengerman, 2017).The adoption of such policies and practices often require significant changes in organizational culture and structure, otherwise known as organizational transformation.

Organizational transformation is a difficult undertaking, as culture and structure are deeply embedded aspects of the organization. Some organizations struggle to value person-centered approaches to enhancing inclusion as opposed to more systemcentered approaches. Others may be challenged to change old ways of doing business, adjust staffing investments, engage employers, or navigate complex licensing systems. Valuing inclusion requires positive 
thinking, learning, creativity, innovation, and continuous quality improvement (Inge, 2008). In addition, organizations need to value supporting individuals in the community and moving beyond facility-based programs. Organizational change is made more complicated by the long history the disability service system has had in providing care and protection for people with disabilities in segregated environments. Relearning, reinvesting, and reinventing an organization that prioritizes individualized, person-centered, and inclusive services can take many years.

There are also other barriers to transformation, including lack of strong internal and external communication plans that include clear expectations for competitive, integrated employment; the need for organization-wide professional development on person-centered practices; the failure to incorporate wrap-around services into plans; and the failure to engage with self-advocates, families, funders, and other community partners (Sulewski et al., 2017).

While organizational transformation is an arduous task, it is not impossible, and there are certain tools and techniques that can enhance efforts toward organizational change. One framework that is useful for organizing those tools and techniques is the model of episodic and continuous change (Weick \& Quinn, 1999). In this framework, episodic change is defined as a one-time change in policy, procedure, training, or other organizational elements. This type of change is focused on short-term adaptation within an organization. The change process is intentionally planned and developed, usually with assistance from experts from outside of the organization. In contrast to episodic change, continuous change is ongoing, evolving change that is constantly occurring and accumulating over time. This change process is responsive to what is happening within the organization and is led by individuals within the organization. It is focused on long-term adaptability within the organization.

Within the context of embracing PCPs and Employment First policies, both types of change are essential. Episodic change is necessary to introduce a shift away from historical or system-focused practices toward the inclusion of PCPs or Employment First policies. This requires instituting organizationwide training and shifts in goals, language, processes, and tools. These episodic changes can happen all at once or at varying times. Regardless of how the process unfolds, there is a clear demarcation between how work was done before the change was implemented and how it is done after the change is implemented. This episodic change is necessary because it introduces a turning point, in which the significant, often practical, changes permeate the whole organization.

If, however, the work stops with episodic change, there will not be true organizational transformation, rather, there will only be superficial change that does not infiltrate the day-to-day work of the organization. For true organizational transformation to take place, in addition to changes within structures, policies, and procedures, each individual employee needs to be able to change his or her mindset and approach to the work of the organization.

In order to move the important work that started with the episodic change forward, the organization must shift to a model of continuous change. Within continuous change, individual members come together in small groups in order to examine processes and practices within the organization, identifying small changes that will allow for further adoption of PCPs or Employment First policies. One way to pursue continuous change is through the development of a community of practice (CoP). This CoP can plan for and analyze different aspects of the organization's operation in order to identify opportunities for advancement.

\section{Case study}

The Institute on Community Integration (ICI) at the University of Minnesota supported a statewide agency that supports competitive, integrated employment for people with disabilities in engaging in organizational transformation that involved the adoption of person-centered thinking (PCT) and person-centered practices (PCP). In the episodic part of this process, ICI staff provided PCT and PCP training for agency staff across the state. These trainings aimed to convey that, in pursuit of a more personcentered service system, services should ensure that individuals with disabilities are treated with dignity and respect, while supporting informed choice and self-direction. Though the use of some personcentered practices, such as Discovery, Individual Placement and Support (IPS), and motivational interviewing (MI) were already be used by certain staff, this organization was aiming to integrate PCT and PCP more thoroughly so that it would permeate all of their interactions with job seekers. Thus, this project provided the opportunity to facilitate the development of the knowledge and skills required for this 


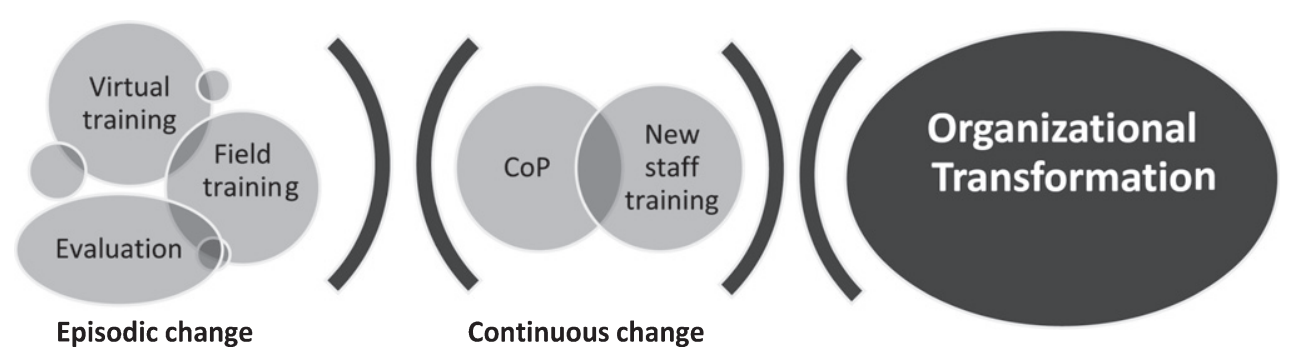

Fig. 1. Case study elements and activities of organizational transformation.

agency's staff to provide robust person-centered services to support job seekers in pursuing competitive, integrated employment.

This project included virtual and in-person training, evaluation, and technical assistance for the development of a CoP. Figure 1 demonstrates how those activities fit within the model of organizational change. These elements were customized for the agency to enhance both organizational and individual transformation toward the adoption of PCPs. Collectively, project activities and deliverables provided the organization with foundational knowledge, best practices, and evidenced-based approaches to incorporate PCPs into the organization's policies and procedures. In addition, evaluation data provided insight into what types of knowledge, skills, and support staff needed to make PCPs part of their everyday approach.

\subsection{Episodic change}

The episodic change portion of the organizational transformation began with a one-day, online introductory training on PCPs delivered via video-conference attended by all organizational staff. This provided all staff with foundational information and a common language while setting the stage for further learning tailored to their unique roles in the organization.

Another one-day training, in-person training was delivered to the organization's administrative team to support them in exploring and developing an organizational culture built around the recognizing and leveraging job seekers' individual strengths and preferences. This training introduced a suite of tools and techniques that support person-centered approaches to help administrative staff grow in their understanding what it would take to support organization-wide adoption of PCPs. This involvement of administrative staff is an example of a central piece of organizational transformation: leadership and staff at all levels within the organization must be invested in and supportive of the necessary change.

Furthermore, ten, one-day, in-person field trainings regarding the use of PCPs and MI in employment and vocational settings were delivered to groups of agency staff in various regions throughout the state. These sessions were intended to support staff in applying the information learned to their specific needs, target populations, and day-to-day work activities.

\subsection{Continuous change: Community of practice}

At the end of the trainings that characterized the preliminary phase of episodic change, continuous change was introduced through the use of an organization-wide CoP. While there are several different ways to structure CoPs and to choose participants for them, this project was focused on promoting the adoption of processes, procedures, language, ongoing training, and policies that would bolster the use of PCPs. As such, the participants in the CoP included individuals throughout the organization who most believed in and were on board with the shift to the adoption of PCPs; these individuals actively chose to be a part of this CoP. Organization leadership invited all staff who were interested in being a part of the CoP to complete an application that included questions regarding their understanding of what needed to be done for the organization to fully adopt PCPs and their motivation to ensure that those things happened. ICI staff provided consultation and technical assistance to support leadership in identifying which individual applicants were most likely to further the effort of the organization to adopt PCPs. In this, there was a particular effort to make sure that the final CoP group included individuals who represented all roles within the organization in order to allow the changes to permeate every level of practice. 
Furthermore, it also included individuals from all of the major geographic locations within the state in order to guarantee that experiences and input from each location were included as the group was further engaging in the process of transformation.

This community of practice had four major roles: to work with response repertoires, engage in improvisation, provide translation, and enhance learning (based on the model from Weick \& Quinn, 1999). By working with response repertoires, members of the CoP worked to enlarge, strengthen, or shrink the possible options for individual behavior within the organization. For example, the CoP considered the intake process, bringing their individual experiences of engaging in the process or watching colleagues engage in the process. They were then able to consider whether there were certain changes to the process that would make it more likely for individual staff members to use PCPs during intake.

As the CoP met as a group, made decisions, and brought up new ideas, the individual members were then able to go back to their work to try new things, use innovative methods, and then report their experiences back to the group. Over time, this type of improvisation allows for organization-wide restructuring based on actual on-the-ground experimentation and feedback. This leads to change through exploration of ongoing variations in practice. For example, members of the $\mathrm{CoP}$ who supervised a group of individuals were able to experiment with integrating PCPs into their supervision model, which had a dual purpose of further integrating personcenteredness into the fabric of the organization while also allowing supervised staff to have a true experience of what it felt like to be on the receiving end of PCPs. These supervisors were then able to bring their experiences back to the CoP group, from which the group was able to consider how to modify the practice or how to integrate it more thoroughly throughout the organization.

The CoP also had the ability to introduce new language and dialogue, and through the adoption of new language, to shift the identity of the organization to have person-centeredness at its core. All experiences and changes happen in the context of language, and the language used can influence and structure that experience. When attempting to make a change at a deep level within the organization, one way to do so is to examine the types of language available and the ways that dialogue can support or inhibit that change. Thus, the CoP had the opportunity to explore current language, try out new ways of talking about things that support a more person-centered view, and build new types of dialogues to bolster that view.

Last, but not least, it is the role of the CoP as a group and as individual members of the organization to engage in learning. In this context, learning is an exploration of how intentional changes can be made in small ways. Within this learning, "each shift in practice creates the conditions for further breakdowns, unanticipated outcomes, and innovations, which are in turn met with more variations" (Orlikowski, 1995, p. 4-5). The CoP provides an incubator in which multiple individuals from the organization are able to work together to process experiences in an effort to continue the organizational transformation process in an ongoing way.

This state-wide agency engaged in a model of organizational transformation that included elements of episodic and continuous change with the goal of creating a shift to a more person-centered culture along with the adoption of PCPs. Throughout this process, the staff at ICI learned important lessons that others can consider when supporting or engaging in organizational change.

\section{Lessons learned}

Episodic change is more dramatic than continuous change, and, as such, it can be more difficult for individuals within an organization to accept. During the course of this project, there was a wide array of opinions of and reactions to the initial training activities, including a bit of resistance. This project began with an organization-wide videoconference, followed by in-person field trainings. In that process, it became clear that initial engagement in organizational transformation is best when it occurs in a face-to-face environment. While virtual training can be cost-effective, it is not necessarily conducive for meaningful engagement, which is a critical aspect of engaging learners in a topic such as PCPs. Between technological difficulties and the impersonal nature of videoconferencing, it was difficult to gauge engagement, understanding, or concerns of individuals within the organization. Were this process to have started with the field trainings, it is possible that the trainers would have been more able to connect directly with the dynamics occurring among the agency's staff. This would have provided a more solid foundation from which to move forward in the organizational change process. It is important to note, however, that, regardless of the format, it is 
reasonable to expect some resistance to episodic change given its abrupt nature.

One strategy that was extremely useful in connecting the episodic and continuous change was to use evaluations from the trainings (i.e., episodic change) as fodder for the CoP group to consider (i.e., continuous change). In this process, ICI staff presented comments from various participant evaluations to the $\mathrm{CoP}$ and led a discussion regarding responses to those comments and how to work with the attitude embedded in the comment in a constructive way. In order to enhance this conversation, ICI staff presented information regarding the stages of change (i.e., The Transtheoretical Model of Behavior Change Prochaska, Norcross, \& DiClemente, 2007) and aided the members of the CoP in determining the stage of change represented by the comment. This allowed the members of the CoP to leverage strategies for working with different stages of change in potential responses to such attitudes within their organization. Within this, CoP members recognized the diversity of opinions, experiences, and perceptions within the agency's staff, which ultimately supported the use of more flexible strategies used to support the continuous adoption and integration of PCPs in the agency's work.

When considering organizational change, it is useful to develop a plan that intentionally includes elements of episodic change and elements of continuous change. These two types of change processes can enhance the likelihood of successful organizational transformation, creating a shift within the organization while supporting ongoing organizational adaptability within the context of that shift.

\section{Conclusion}

PCPs and employment-first policies can serve as facilitators for increase the rates of competitive, integrated employment for people with disabilities; however, many local and state organizations find it difficult to shift gears to focus on competitive, integrated employment and individualized supports. PCPs can provide a foundation from which to develop and refine policies, procedures, and activities that focus on the individual job seeker; however, the adoption of PCPs can require a significant investment in organizational transformation. With the an intentional combination of episodic and continuous change activities, organizations can effectively and efficiently engage in this type of organizational transformation to increase community participation and employment for people with disabilities.

\section{Acknowledgments}

This manuscript is a product of the Rehabilitation Research and Training Center on Community Living (RTC-CL) at the University of Minnesota, funded by the National Institute on Disability, Independent Living, and Rehabilitation (Grant number HHS-2018-ACLNIDILRR-RTCP-0262). NIDILRR is a Center within the Administration for Community Living (ACL), Department of Health and Human Services (HHS), The contents of this publication do not necessarily represent the policy of NIDILRR, ACL, HHS, and you should not assume endorsement by the Federal Government.

\section{Conflict of interest}

None to report.

\section{References}

Gunty, A. L., Dean, K., Nord, D., Hoff, D., \& Nye-Lengerman, K. (2017). Employment First: An update on national progress. Minneapolis, MN.

Inge, K. (2008). Customized Employment. Journal of Vocational Rehabilitation, 28, 133-134.

Orlikowski, W. J. (1995). Improvising organizational transformation over time: A situated change perspective. Information Systems Research, (September), 1-57.

Prochaska, J., Norcross, J. C., \& DiClemente, C. C. (2007). Changing for good: A revolutionary six-stage program for overcoming bad habits and moving your life positively forward. New York, New York: HarperCollins Publishing.

Sulewski, J. S., Ciulla, J. C., Lyons, O., Lucas, J., Vogt, T., \& Bachmeyer, K. (2017). Organizational transformation to integrated employment and community life engagement. Journal of Vocational Rehabilitation, 46, 313-320. https://doi.org/10.3233/JVR-170867

Weick, K. E., \& Quinn, R. E. (1999). Organizational change and development. Annual Review of Psychology, 50, 361-386. https://doi.org/10.1146/annurev.psych.50.1.361 